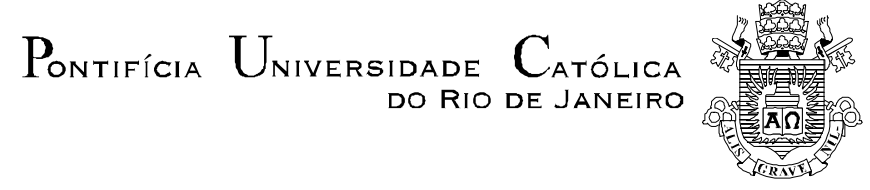

Rafael Garcia Dutra

\title{
Gerenciamento de Risco em Empresas Não Financeiras: \\ Aplicações na Indústria Sucroenergética
}

\begin{abstract}
Dissertação de Mestrado
Dissertação apresentada como requisito parcial para obtenção do grau de Mestre pelo Programa de Pós-Graduação em Engenharia de Produção da PUC-Rio.
\end{abstract}

Orientador: Prof. Carlos Patricio Samanez 
Pontifícia Universidade Católica $_{\text {a }}$

\section{Rafael Garcia Dutra}

\section{Gerenciamento de Risco em Empresas não Financeiras: Aplicações na indústria Sucroenergética}

Dissertação apresentada como requisito parcial para obtenção do grau de Mestre pelo Programa de Pós-Graduação em Engenharia de Produção da PUC-Rio. Aprovada pela Comissão Examinadora abaixo assinada.

Prof. Carlos Patrício Samanez Orientador Departamento de Engenharia Industrial - PUC-Rio

Prof. José Paulo Teixeira Departamento de Engenharia Industrial - PUC-Rio

Prof. Fabio Rodrigo Siqueira Batista Departamento de Engenharia Industrial - PUC-Rio

Prof. José Eugenio Leal Coordenador Setorial do Centro Técnico Científico - PUC-Rio 
Todos os direitos reservados. É proibida a reprodução total ou parcial do trabalho sem autorização da universidade, da autora e do orientador.

\section{Rafael Garcia Dutra}

Graduado em Administração de Empresas pela Pontifícia Universidade Católica - PUC Rio em Novembro de 2008. Depois de concluído o curso, ingressou no mestrado de Engenharia de Produção do Departamento de Engenharia Industrial da PUC Rio.

Ficha Catalográfica

Dutra, Rafael Garcia

Gerenciamento de risco em empresas não financeiras: aplicações na indústria sucroenergética / Rafael Garcia Dutra ; orientador: Carlos Patrício Samanez. -2011.

76 f. : il. (color.) ; $30 \mathrm{~cm}$

Dissertação (mestrado)-Pontifícia

Universidade Católica do Rio de Janeiro, Departamento de Engenharia Industrial, 2011.

Inclui bibliografia

1. Engenharia Industrial - Teses. 2. Fatores de risco. 3. Empresas não-financeiras. 4. Fluxo de caixa. 5. Risco de mercado. I. Samanez, Carlos Patricio. II. Pontifícia Universidade Católica do Rio de Janeiro. Departamento de Engenharia Industrial. III. Título.

CDD: 658.5 


\section{Agradecimentos}

Agradeço aos meus pais, Affonso Dutra e Rosana Dutra, por todo o suporte intelectual e motivacional que me forneceram durante o mestrado.

Aos professores do Departamento de Engenharia Industrial, especialmente o Carlos Patricio Samanez, meu orientador, e o professor Tara Baidya.

Agradeço a CAPES, pelo auxilio financeiro.

À Marcela Bejarano, por escutar e discutir todos os pontos levantados por mim durante o curso.

Ao Eduardo Lage e à Priscilla Pessoa, meus grandes companheiros no decorrer desta etapa. 


\section{Resumo}

Dutra, Rafael Garcia; Samanez, Carlos Patricio. Gerenciamento de Risco em Empresas Não Financeiras: Aplicações na Indústria Sucroenergética. Rio de Janeiro, 2011. 76p. Dissertação de Mestrado Departamento de Engenharia de Produção. Pontifícia Universidade Católica do Rio de Janeiro.

O gerenciamento de Risco para empresas não financeiras é de grande importância, e vem recebendo cada vez mais relevância no mercado financeiro no país. O risco de mercado, ou seja, a volatilidade das variáveis as quais as companhias estão submetidas deve ser analisada com extrema atenção, dado que estas variáveis impactam de maneira significativa os fluxos de caixa e o valor destas. Esta pesquisa tem como objetivo estudar a utilização do Fluxo de Caixa em Risco (Cash Flow at Risk - CfaR), um instrumento para controle de risco de mercado que simula o valor em risco do fluxo de caixa futuro de uma companhia dentro de um intervalo de confiança predefinido. Faremos uma apresentação do modelo CfaR em uma empresa não financeira, em particular uma companhia do setor Sucroenergético para se estimar a probabilidade desta empresa não possuir a liquidez necessária para arcar com seus compromissos financeiros de curto prazo.

\section{Palavras-Chave}

Fatores de Risco; Empresas não-financeiras; Fluxo de Caixa; Risco de Mercado. 


\section{Abstract}

Dutra, Rafael Garcia; Samanez, Carlos Patricio (Advisor). Risk Management in Non-Financial Companies: Applications to the Sucroenergetic Sector. Rio de Janeiro, 2011. 76p. MSc. Dissertation Departamento de Engenharia Industrial. Pontifícia Universidade Católica do Rio de Janeiro.

Risk Management for non financial companies is of great importance and has been gaining even more significance in the country's financial market. The Market Risk, that is, the volatility of the variables in which companies are exposed has to be analyzed with extreme attention, given that such variations may impact strongly the companies' cash flows and its values. This paper has the aim to study the use of the Cash Flow at Risk - CfaR, a tool to control market risk and that simulates the Value at Risk of the future cash flow of a company at a predetermined confidence interval. The goal is to make a presentation of the CfaR model to a non financial company, particularly a company in the Sucroenergetic sector to estimate the probability of this specific enterprise not having the liquid assets necessary to honor its short term commitments.

\section{Keywords}

Risk Factors; Non Financial Companies; Cash Flow; Market Risk. 


\section{Sumário}

1 Introdução 10

1.1. Revisão bibliográfica 12

1.2. Justificativa do trabalho 15

$\begin{array}{ll}\text { 1.3. Pergunta a ser respondida } & 16\end{array}$

1.4. Metodologia 16

$\begin{array}{ll}\text { 1.5. Descrição dos capítulos } & 18\end{array}$

2 Risk Management e Governança Corporativa 19

2.1. Mecanismos de Governança Corporativa 19

2.2. Adicionando Valor a Firma com Risk Management 21

3 Métricas para Gerenciamento de Riscos em Empresas Não

Financeiras $\quad 24$

3.1. Value at Risk (VaR) 24

3.2. Cash Flow at Risk (CfaR) e a Metodologia CorporateMetrics 27

3.2.1. Especificação da Medida de Risco 27

3.2.1.1. Cash Flow at Risk (CfaR) 28

3.2.1.2. Earnings At Risk (EaR) 29

3.2.2. Mapeamento de Exposições 30

3.2.3. Geração de Cenários 30

3.2.4. Avaliação 30

$\begin{array}{ll}\text { 3.2.5. Cálculo do Risco } & 30\end{array}$

4 Processos Estocásticos e Simulação de Monte Carlo 33

4.1. Processos de Wiener 33

4.1.1. Movimento Browniano com Drift 34

4.1.2. Movimento Geométrico Browniano 35

4.2. Simulação de Monte Carlo 37

5 O Setor Sucroenergético 40

5.1. O Setor Sucroenergético no Brasil 40

5.1.1. Cana de Açúcar na Produção do Açúcar 41 
5.1.2. Cana de Açúcar na Produção de Etanol e Energia Elétrica (Bioeletricidade Sucroenergética)

5.2. O Setor Sucroenergético no Mundo

5.2.1. Açúcar

5.2.2. Etanol e Bioeletricidade

6 A empresa e o Estudo de Caso

6.1. Perfil Corporativo

6.2. Estrutura Empresarial

6.3. Produtos e Subprodutos

6.4. Localização 49

6.5. Capacidade de Moagem de Cana de Açúcar 49

6.6. Investimentos Relevantes $\quad 50$

6.7. Breve Histórico 51

6.8. Estrutura Adotada 53

6.9. Síntese da Métrica 59

7 Conclusão 62

8 Referências Bibliográficas $\quad 64$

9 Anexos $\quad 71$

9.1. Anexo A - Desempenho de Vendas São Martinho S.A 71

9.2. Anexo B - Demonstração do Resultado São Martinho S.A 72

9.3. Anexo C - Fluxo de Caixa Consolidado 3T11 73

9.4. Anexo D - Fluxo de Caixa Consolidado 2T11 74

9.5. Anexo E - Fluxo de Caixa Consolidado 1T11 75

9.6. Anexo F - Fluxo de Caixa Consolidado Ano Fiscal 2010 


\section{Lista de Tabelas}

Objetivos e Procedimentos dos 5 Passos Corporate

Tabela 1 Metrics

Tabela 2 Resultado dos 5 Passos Corporate Metrics 30

Tabela 3 Evolução do Volume de Produção no Brasil 40

Tabela 4 Bioeletricidade - Volume Comercializado (Mwméd) 41

Evolução do Volume de Produção do Grupo São

Tabela 5 Martinho 48

$\begin{array}{lll}\text { Tabela } 6 & \text { Premissas } & 51\end{array}$

Tabela 7 Volumes Históricos $\quad 52$

Tabela 8 Volumes Projetados 52

Tabela 9 Matriz de Correlações $\quad 55$

Tabela 10 Parâmetros do MGB 56

$\begin{array}{lll}\text { Tabela } 11 \text { Estatísticas de Risco } & 58\end{array}$

\section{Lista de Figuras}

Figura 1 Scenario Tool - Dados Históricos imputados 15

Figura 2 Scenario Tool-Geração dos cenários 16

Figura 3 Distribuição de freqüência do lucro de uma empresa hipotética 25

Figura 4 Açúcar - Principais Produtores Safra 2008/09 (Milhões de Toneladas - Raw Sugar) 43

Figura 5 Etanol - Principais Produtores 2008 (Bilhões de Litros) 44

Figura 6 Fluxo de Caixa Proposto 53

Figura 7 Gráfico Tornado $\quad 54$

Evolução dos preços das variáveis no período

Figura 8 analisado 56

$\begin{array}{lll}\text { Figura } 9 \text { Distribuição do Fluxo de Caixa } & 58\end{array}$ 